\title{
Complementary and alternative medicine use amongst Malaysian orthopaedic oncology patients
}

Amreeta Dhanoa ${ }^{{ }^{*}}$, Tze Lek Yong ${ }^{1}$, Stephanie Jin Leng Yeap ${ }^{1}$, Isaac Shi Zhung Lee ${ }^{1}$ and Vivek Ajit Singh ${ }^{2}$

\begin{abstract}
Background: Although studies have shown that a large proportion of cancer patients use CAM, no study on CAM use amongst orthopaedic oncology patients has been published. Therefore, this study aims to determine the prevalence, characteristics and factors associated with CAM use amongst orthopaedic oncology patients.

Methods: All consecutive consenting patients/parents who presented at the Orthopaedic Oncology Clinic, University Malaya Medical Centre (1st January to 31st December 2013) were interviewed using a structured questionnaire.

Results: Overall, one hundred sixty-eight of the 274 patients recruited (61.3\%) had used CAM at some time during their current illness. The prevalence of CAM used was 68\% (123/181) for patients with malignant tumours and 48.4\% (45/93) for patients with benign tumours. The most popular CAMs were biological-based therapies (90.5\%), followed by mind-body techniques (40.5\%). The most frequently used biological therapies were mega/multivitamins (31\%), snakehead (Chana striatus) (28\%) and sea cucumber (Stichopus horrens) (18\%); whereas prayers (31\%) and holy water (13\%) dominated the mind-body category. Common reasons for CAM use were to improve physical well-being (60.1\%), try out everything that would help (59.5\%) and to enhance wound-healing (39.3\%). Independent predictors for CAM use in multivariate analysis were paediatric patients [OR 2.46; 95\% Cl 0.99-6.06; $p=0.05$ ], malignant tumours [OR 1.90; 95\% Cl 1.12-3.25; $p=0.018$ ] and patients who underwent surgery [OR 2.06; 95\% Cl 1.15-3.69; $p=0.015$ ]. Majority patients started taking CAMs following suggestions from family members (53\%) and friends (49\%). Sixty-six percent of patients felt they actually benefitted from CAM and 83.3\% were satisfied/very satisfied. Only 5 patients reported side-effects. Majority of CAM users planned to continue CAM use or recommend it to others. However, only $31.5 \%$ of patients disclosed their CAM usage to their doctors.
\end{abstract}

Conclusions: This survey revealed a high prevalence of CAM usage amongst orthopaedic oncology patients, with majority patients expressing satisfaction towards CAM. Oncologists should proactively ask patients about CAM to prevent potential adverse effects, as most patients do not share this information with them.

Keywords: Complementary medicine, Alternative, Orthopaedic, Oncology, Cancer, Tumour, CAM

\footnotetext{
*Correspondence: amreeta.dhanoa@monash.edu

'Jeffrey Cheah School of Medicine and Health Sciences, Monash University, 47500 Bandar Sunway, Malaysia

Full list of author information is available at the end of the article
} 


\section{Background}

The desire among human beings to explore beyond the realms of conventional medical treatment is clearly illustrated by the usage of complementary and alternative medicine (CAM) during illness [1,2]. This usage is more pronounced when patients have difficult to treat chronic medical ailments such as chronic pain, poor mental health and cancer for which conventional medicine may not provide effective and satisfactory remedies [1,2]. Cancer ranks amongst the most dreaded of all illness [3]. As the diagnosis, symptoms and treatment of cancer challenges every dimension of a person's life including physical, emotional, mental and spiritual aspects [3], patients with cancer are more receptive to CAM utilization. CAM are defined as medical and healthcare systems, practices, and products that are not currently considered an integral part of conventional medicine [4].

Many CAM users report use not so much as a result of being dissatisfied with conventional medicine, but largely because they found these healthcare alternatives to be more congruent with their own values, beliefs, and philosophical orientations toward health and life [1]. Although the effectiveness and safety of certain CAM are questionable, studies have reported widespread CAM usage amongst cancer patients to increase body's ability to fight cancer, improve quality of life, strengthen immune system and cope with disease symptoms [1,5-7]. Others use CAM as a last resort and as a way of finding hope $[3,7,8]$. Essentially, CAM meets the demands that mainstream medicine cannot principally satisfy, and provides complementary solutions to conventional medicine [9].

The increasing popularity of CAM amongst cancer patients is well recognized. A recent European survey reported that the overall prevalence of CAM used by cancer patients was $35.9 \%$, ranging from $14.8 \%$ to $73.1 \%$ [5]. There has been a noticeable increase in CAM usage amongst cancer patients, from $25 \%$ in the $1970-1980$ s to $32 \%$ in the 1990s and 49\% after 2000 [10]. As cancer incidence increases and survival time lengthens, patients using CAM are likely to escalate [7]. This trend is gradually recognized by oncologists, although not many patients discuss these therapies with them [7].

Evidence-based recommendations regarding the usefulness and safety or lack thereof of CAM practices in oncology setting continues to evolve [11-17]. An increasing number of evidence-based studies support the use of CAM therapies as a complement to conventional cancer therapies. For instance, Chinese medicinal herbs have been shown to reduce treatment side-effects, improve quality of life and survival rates amongst adult cancer patients [11]. Hypnosis lessens pain, anxiety and distress during procedures and appears to be especially effective amongst paediatric cancer patients [12]. Medical Qigong has been shown to improve quality of life, mood and fatigue and reduces inflammation amongst cancer patients [13]. Despite the growing scientific evidence favoring CAM usage, numerous reports highlighting the adverseeffects and risks to patient safety resulting from direct effect or possible interactions with conventional therapies are emerging [14]. St John's wort reduces plasma levels of the active metabolites of three chemotherapy agents: irinotecan, imatinib mesylate, and docetaxel, with potential deleterious consequences on treatment outcome [15]. Although kava-containing products were found to be effective in controlling anxiety, stress and insomnia, recent reports associate this herbal remedy with severe hepatotoxicity [16]. Garlic, ginkgo, and ginseng, have been associated with postoperative bleeding via platelet inhibitory effects [17].

To date, numerous studies highlighting the growing use of CAM amongst cancer patients have been reported. These studies report either on patients with wide arrays of cancers or focuses on specific cancer particularly breast, prostate, gynecological and colorectal cancers [8]. However, to our best knowledge, this study is the first to specifically explore CAM usage amongst patients with orthopaedic oncology tumours. Another important distinction regarding this study is that it involves patients of all age groups and encompasses both malignant and benign tumours. This is unlike most other studies that involve either adults or children and focus solely on malignant tumours [6-8,10,18-20]. Advances in treatment in paediatric oncology field, have resulted in an increase of 5-year survival rate of patients to approximately $80 \%$ [21]. One important consequence of this paradigm shift is that survivors of childhood cancer are likely to encounter acute and long-term adverse-effects resulting from the illness or mainstream medical therapies [22]. This drives many parents and patients to use CAM to manage disease symptoms, treatment side-effects and repeated painful operations despite the paucity of data supporting their efficacy and safety [12,22].

Thus, an exhaustive coverage is integral towards understanding CAM usage amongst orthopaedic oncology patients, since this field comprises both malignant and benign tumours and involves all age-groups. Therefore, this study was conducted amongst patients with orthopaedic oncology tumours to determine: the prevalence, characteristics, predictors and reasons for CAM use, types of CAM therapies and practices used, satisfaction with CAM, source of information and disclosure to doctors.

\section{Methods}

Study design, setting and study population

This was a prospective, cross-sectional study conducted from 1st January to 31st December, 2013 at the Orthopaedic Oncology Clinic, University Malaya Medical Centre (UMMC), a 1051-bedded tertiary referral centre situated in Kuala Lumpur. As the leading referral 
centre for orthopaedic oncology patients in Malaysia, its patient population is reflective of a larger community in Malaysia. The study was approved by the Medical Ethics Committee of UMMC (Reference No: 944.12).

Patients with histologically confirmed bone and soft tissue tumours, diagnosed for at least 2 months were recruited consecutively as they presented to the clinic. Informed consent was obtained from patients or parents/ caregivers for patients $<18$ years. Patients were excluded if they were unable to give informed consent or if their condition precluded the ability to do so.

\section{Questionnaire}

For questionnaire development, an extensive literature search exploring CAM usage amongst cancer patients was conducted. Pre-test of the questionnaire was undertaken to obtain feedback from 6 orthopaedic oncology patients. Patients were asked to suggest CAMs not already in the existing list. The pre-test feedback and information captured from healthcare professionals, was used to develop a culturally relevant and comprehensive questionnaire. Four interviewers were trained to administer the questionnaires. To increase reliability and validity of responses, face-to-face interviews were conducted using structured questionnaire.

The questionnaire comprised three main sections. The first section captured basic socio-demographic data, such as age, gender, religion, marital status, highest education level and income. The second section captured diseaserelated characteristics such as tumour type, presence of metastasis, time since diagnosis and treatment. This information was cross-checked with medical notes. Bone and soft tissue tumours were classified according to World Health Organization (WHO) classification [23].

Each patient was presented with a list of CAMs on the questionnaire. These CAMs were broadly divided into 5 modalities based on National Centre for Complementary and Alternative Medicine (NCCAM, 2005) and Cochrane CAM Field categorization [24,25]. These were: biological, spiritual/mind-body, alternative, physical (body-based) and energy therapies. Patients were then asked if they had used any of the listed CAMs or any other CAMs not listed, either before or after their diagnosis. Patients were considered CAM users if they had used CAM at least once and non-users if they had not used any CAM during the current illness. Patients who had been using CAM even before their diagnosis were regarded non-users, unless a change in pattern of usage was demonstrated after diagnosis, e.g. increased dose or frequency. The third section of the questionnaire captured information on types of CAMs, reasons for use, benefits attained, satisfaction, source of information and disclosures to doctors. For paediatric patients, the questionnaire was answered mainly by their parents. Non-users were acknowledged after completing the socio-demographic and clinical section and their interview ended here.

Data was analysed using SPSS version 20.0, comparing CAM users and non-users. Categorical variables were compared using Fisher's exact test or Chi-squared test $(p<0.05)$. Variables associated with CAM usage in the univariate analysis were entered into multivariate logistic regression analysis. Where appropriate, CAM use was compared in patients with malignant verses benign tumours and in adult verses paediatric patients.

\section{Results}

Prevalence, clinical characteristics and socio-demographic status

During the one year study period, 285 patients were interviewed, with complete data obtained from 274 patients. There were 181 (66.1\%) patients who presented with malignant tumours and 93 (33.9\%) with benign tumours. The various bone and soft tissue tumours (WHO, 2013) [23] are presented in Table 1 . Mean age was $41.27 \pm 20.53$ years (range 8 to 90 years).

Overall, 168 patients (61.3\%) had used CAM at some time during their current illness. The prevalence of CAM used was 68\% (123/181) for patients with malignant tumours and $48.4 \%$ (45/93) for patients with benign tumours. Overall, $75.2 \%$ patients underwent surgery, $26.6 \%$ underwent chemotherapy and 18.6\% radiotherapy. Majority patients used CAM either concurrently with conventional therapy $(44 \%)$ or upon completion of conventional treatment (44.6\%); whereas $11.3 \%$ started CAM before conventional therapy but stopped upon commencing conventional treatment.

\section{Predictors for using CAM}

Factors associated with CAM usage in univariate analyses $(\mathrm{p}<0.05)$ (Table 2) were paediatric patients, malignant tumours, metastasis, patients who underwent chemotherapy and surgery. Multivariate analysis revealed that paediatric patients (OR 2.46; 95\% CI 0.99-6.06; $\mathrm{p}=0.05$ ), malignant tumours (OR 1.90; 95\% CI 1.12-3.25; p = 0.018) and surgery (OR 2.06; 95\% CI 1.15-3.69; $\mathrm{p}=0.015)$ remained significant predictors. CAM use was not affected by gender, ethnicity, marital status, income, educational level and employment status.

\section{Reasons for using CAM}

Overall, the most common reasons that drove patients to utilize CAM were to enhance overall health and physical well-being (60.1\%), to try out everything that would help (59.5\%) and to enhance wound healing (39.3\%) (Table 3). When analysed separately, patients with malignant tumours cited the first two reasons more commonly whereas enhancement of wound healing was more common amongst patients with benign tumours. Other reasons 
Table 1 Histological diagnosis (WHO Classification, 2013) [23] $(n=274)$

\begin{tabular}{|c|c|}
\hline \multicolumn{2}{|l|}{ Malignant tumours of Bone and Soft Tissue $(\mathrm{N}=181)$} \\
\hline \multicolumn{2}{|l|}{ Bone $(n=109)$} \\
\hline Osteogenic tumours $^{\mathrm{a}}$ & 37 \\
\hline Metastasis to bone & 33 \\
\hline Miscellaneous tumors ${ }^{\mathrm{b}}$ & 17 \\
\hline Chondrogenic tumours $^{c}$ & 13 \\
\hline Haematopoietic neoplasms & 8 \\
\hline Tumours of undefined neoplastic nature & 1 \\
\hline \multicolumn{2}{|l|}{ Soft tissue $(n=72)$} \\
\hline Undifferentiated/unclassified sarcoma & 22 \\
\hline Adipocytic tumours & 17 \\
\hline Tumors of uncertain differentiation & 16 \\
\hline Fibroblastic/myofibroblastic tumors & 8 \\
\hline Nerve sheath tumor & 4 \\
\hline Smooth muscle tumors & 3 \\
\hline Vascular tumors of soft tissue & 1 \\
\hline Skeletal muscle tumors & 1 \\
\hline
\end{tabular}

Benign tumours of Bone and Soft Tissue $(n=93)$

Bone $(n=55)$

Osteoclastic giant cell rich tumors

Tumor of undefined neoplastic nature

Chondrogenic tumor

Osteogenic tumor

Soft tissue $(n=38)$

Adipocytic tumor
Vascular tumor of soft tissue
Fibroblastic/Myofibroblastic tumor
Nerve sheath tumor
Fibrohistocytic tumor
Benign tumor of uncertain differentiation

${ }^{a}$ All were osteosarcoma, ${ }^{6} 64.7 \%$ were Ewing sarcoma, ${ }^{\mathrm{C}} \mathrm{All}$ were chondrosarcoma.

did not differ significantly between the two groups and included controlling disease progression (32.1\%), boosting immune system (27.4\%) and alleviating disease symptoms and toxic effects of conventional therapy (26.2\%).

We then compared the paediatric and adult oncology patients to determine the CAM motivators, and noted no significant differences (Table 3). Most parents indicated that they wanted to do everything possible for their child (60\%) and to improve the child's general-health (56\%), paralleling the main reasons cited amongst the adult patients. However, reasons like controlling disease progression, seeking a cure, boosting immune system, alleviating side-effective of conventional therapy were notably higher amongst the paediatric patients, although not statistically significant. It was reassuring to note that only 4 patients utilized CAM because they felt dissatisfied with conventional therapy.

\section{Types of CAM used}

The most common CAM modalities used were biologicalbased therapies (90.5\%), followed by mind-body techniques $(40.5 \%)$ (Table 4). Patients with malignant tumours were more likely to use biological-based therapies $(p=0.04)$. Conversely, patients with benign tumours were more likely to use physical-based therapies (0.002). Majority patients $(61.5 \%)$ had used 2 or more CAM modalities. When paediatric and adult patients were compared (Table 4), biological therapies were used with equal frequencies and were the most commonly used CAMs amongst both the groups. In contrast, alternative therapy usage was significantly higher amongst the adults patients $(\mathrm{p}=0.027)$. Although mindbody and physical practices were notably higher in adult patients, the difference was not statistically significant.

The various CAMs used within each modality are detailed in Table 5 . Most frequently used biological therapies were mega/multivitamins (31\%), snakehead (Chana striatus) (28\%), sea cucumber (Stichopus horrens) (18\%), herbal remedies (13\%), fish oil (13\%), fruits juices with antioxidant properties (10\%), spirulina (9\%), and sabah snake grass (Clinacanthus nutans) (7.7\%). Mega/multivitamins were used in combination with other CAMs in all but one patient.

The most common mind-body techniques were prayers (31\%) and holy water (13\%), whereas, various message techniques (19.6\%) dominated the physical therapy category. After excluding prayer healing, the overall CAM prevalence remained almost the same (60\%), as almost all patients used other CAM modalities alongside prayer healing. Other popular CAM methods were acupuncture (9.5\%), traditional healers (5.4\%) and various traditional Chinese medicines (6\%). The most common CAMs used amongst the 206 patients who underwent surgery were snakehead and multivitamins/megavitamins $(21.36 \%$ each respectively) followed by sea cucumber (9.7\%).

Amongst the paediatric oncology patients, multi/megavitamins were the most frequently used CAM (56\%). Other biological-based therapies commonly used amongst children were spirulina (20\%), freshwater eel (16\%), and antioxidant pills (16\%). Mind-body medicine (28\%) was the second most common CAM modality used; (prayer/ faith healing, $\mathrm{n}=5$, holy water, $\mathrm{n}=5$ and traditional healer, $n=1$ ). On the contrary, various physical and energy therapies seemed unpopular amongst paediatric oncology patients; only 4 patients used them (massage, $\mathrm{n}=2$, cupping, $\mathrm{n}=1$, bioelectromagnetic therapy, $\mathrm{n}=1$ ). Conspicuously, none of the alternative therapies were used by the paediatric oncology patients; all 16 patients who used acupuncture were adult patients. 
Table 2 Predictors of CAM use

\begin{tabular}{|c|c|c|c|c|c|c|c|}
\hline \multirow{2}{*}{$\frac{\text { Variable }}{\text { Paediatric }}$} & \multicolumn{2}{|c|}{ All patients $(\mathrm{N}=274)(\%)$} & \multirow{2}{*}{$\begin{array}{l}\text { Users of CAM N (\%) } \\
25(78.1)\end{array}$} & \multirow{2}{*}{$\begin{array}{l}\text { Nonusers of CAM N (\%) } \\
7(21.9)\end{array}$} & \multirow{2}{*}{$\begin{array}{l}\mathbf{P} \\
0.04\end{array}$} & \multirow{2}{*}{$\frac{\text { OR }}{2.47}$} & \multirow{2}{*}{$\begin{array}{l}\mathbf{9 5 \%} \mathrm{C1} \\
1.03-5.92\end{array}$} \\
\hline & Yes & $32(11.7)$ & & & & & \\
\hline & No & $242(88.3)$ & $143(59.1)$ & $99(40.9)$ & & & \\
\hline \multirow[t]{4}{*}{ Age range } & $<20$ & $58(21.2)$ & $38(65.5)$ & $20(34.5)$ & 0.43 & & \\
\hline & $21-40$ & $77(28.1)$ & $43(55.8)$ & $34(44.2)$ & & & \\
\hline & $41-60$ & $78(28.5)$ & $52(66.7)$ & $26(33.8)$ & & & \\
\hline & $>60$ & $61(22.3)$ & $35(57.4)$ & $26(42.6)$ & & & \\
\hline \multirow[t]{2}{*}{ Gender } & Male & $129(47.1)$ & $73(56.6)$ & $56(43.4)$ & 0.13 & 1.46 & $0.89-2.38$ \\
\hline & Female & $145(52.9)$ & $95(65.5)$ & $50(34.5)$ & & & \\
\hline \multirow[t]{3}{*}{ Race } & Malay & $118(43.1)$ & $76(64.4)$ & $42(35.6)$ & 0.64 & & \\
\hline & Chinese & $109(39.8)$ & $65(59.6)$ & $44(40.4)$ & & & \\
\hline & Indians & $47(17.2)$ & $27(57.4)$ & $20(42.6)$ & & & \\
\hline \multirow[t]{2}{*}{ Marital status* } & Married & $163(67.4)$ & $101(62.0)$ & $62(38.0)$ & 0.19 & 1.44 & $0.83-2.47$ \\
\hline & Not married & 79 (32.6) & $42(53.2)$ & $37(46.8)$ & & & \\
\hline \multirow[t]{2}{*}{ Education* } & Primary/secondary & $167(69.0)$ & $94(56.3)$ & $73(43.7)$ & 0.19 & 1.46 & $0.83-2.58$ \\
\hline & College/university & $75(31.0)$ & $49(65.3)$ & $26(34.7)$ & & & \\
\hline \multirow[t]{2}{*}{ Job status* } & Employed & $109(45.0)$ & $61(56.0)$ & $48(44.0)$ & 0.37 & 1.27 & $0.76-2.12$ \\
\hline & Unemployed & $133(55.0)$ & $82(61.7)$ & $51(38.3)$ & & & \\
\hline \multirow[t]{2}{*}{ Income* } & Less than 1000 & $151(62.4)$ & $91(60.3)$ & $60(39.7)$ & 0.63 & 1.14 & $0.67-1.93$ \\
\hline & More than 1000 & $91(37.6)$ & $52(57.1)$ & $39(42.9)$ & & & \\
\hline \multirow[t]{2}{*}{ Presence of metastasis } & Yes & $88(32.1)$ & $62(70.5)$ & $26(29.5)$ & 0.03 & 1.80 & $1.05-3.10$ \\
\hline & No & $186(67.9)$ & $106(57.0)$ & $80(43.0)$ & & & \\
\hline \multirow[t]{2}{*}{ Malignancy } & Malignant & $181(66.1)$ & $123(68.0)$ & $58(32.0)$ & 0.002 & 2.26 & $1.36-3.78$ \\
\hline & Benign & $93(33.9)$ & $45(48.4)$ & 48 (51.6) & & & \\
\hline \multirow[t]{2}{*}{ Chemotherapy } & Yes & $73(26.6)$ & $55(75.3)$ & $18(24.7)$ & 0.004 & 2.38 & $1.31-4.34$ \\
\hline & No & $201(73.4)$ & $113(56.2)$ & $88(43.8)$ & & & \\
\hline \multirow[t]{2}{*}{ Surgery } & Yes & $206(75.2)$ & $137(66.5)$ & $69(33.5)$ & 0.002 & 2.37 & $1.36-4.14$ \\
\hline & No & $68(24.8)$ & $31(45.6)$ & $37(54.4)$ & & & \\
\hline \multirow[t]{2}{*}{ Radiotheraphy } & Yes & $51(18.6)$ & 35 (68.6) & $16(31.4)$ & 0.24 & 1.48 & $0.77-2.83$ \\
\hline & No & $233(81.4)$ & $133(59.6)$ & $90(40.4)$ & & & \\
\hline
\end{tabular}

$\mathrm{N}=274$ for all variables, except variables labelled $*(\mathrm{~N}=242)$ as variable* excludes paediatric patients.

\section{Satisfaction with CAM}

Overall, majority of CAM users were very satisfied/satisfied $(83.3 \%)$ with no significant differences in CAM satisfaction rates between malignant and benign tumour group $(\mathrm{p}=0.1)$ In response to an open-ended question, $66 \%$ of CAM users felt they actually experienced positive effects which were feeling more energetic and stronger (45\%), enhanced wound healing $(22.5 \%)$, pain relief $(18 \%)$, calmer mind and better sleep (11.7\%), tumour shrinkage $(5.4 \%)$, improved appetite (5.4\%), fewer side-effects from chemotherapy (3.6\%), internal cleansing and body detoxification (3.6\%) and suppression of disease progression (2.7\%). Majority of CAM users (67\%) planned to continue CAM use or recommend it to others. Similarly, majority parents (84\%) were satisfied with CAM use and 64\% felt that their child had actually benefitted in some way.
Only 5 patients ( 4 adults and 1 child) experienced unwanted effects which were; enlargement of swelling after traditional message $(n=2)$, facial swelling after consuming Chinese medication $(n=1)$, heatiness after consuming mushroom- based capsules $(n=1)$ and excessive coughing after consuming sabah snake grass $(n=1)$. Only 3 patients discontinue conventional medicine for CAM; the reasons cited were cost, side-effects and fear of radiotherapy.

\section{Source of information and disclosure}

When asked how CAM modalities were selected, majority indicated family members (53\%) and friends (49\%) as their main source, followed by mass media/websites (24\%) and own-will (18.5\%). Only $14.3 \%$ of CAM usage was based on recommendations by healthcare personnel (Table 6). When analysed separately, the only difference 
Table 3 Reasons for CAM use amongst 168 CAM users

\begin{tabular}{|c|c|c|c|c|c|c|c|}
\hline Reasons for using CAM & $\begin{array}{l}\text { All patients } \\
(\mathrm{N}=168)(\%)\end{array}$ & $\begin{array}{l}\text { Malignant } \\
(\mathrm{N}=123)(\%)\end{array}$ & $\begin{array}{l}\text { Benign } \\
(\mathrm{N}=45)(\%)\end{array}$ & $P^{1}$ & $\begin{array}{l}\text { Adults } \\
(\mathrm{N}=143)(\%)\end{array}$ & $\begin{array}{l}\text { Paediatric } \\
(\mathrm{N}=25)(\%)\end{array}$ & $\mathrm{P}^{2}$ \\
\hline Enhance overall health/physical well-being & $101(60.1)$ & $80(65.0)$ & $21(46.7)$ & 0.03 & $87(60.8)$ & $14(56.0)$ & 0.648 \\
\hline Trying everything that can help & $100(59.5)$ & $79(64.2)$ & $21(46.7)$ & 0.04 & $85(59.4)$ & $15(60.0)$ & 0.958 \\
\hline Enhance wound healing & $66(39.3)$ & $42(34.1)$ & $24(53.3)$ & 0.02 & $58(40.6)$ & $8(32.0)$ & 0.419 \\
\hline Control disease progression & $54(32.1)$ & $39(31.7)$ & $15(33.3)$ & 0.84 & $44(30.8)$ & $10(40.0)$ & 0.362 \\
\hline Boost immune system to fight disease & $46(27.4)$ & $36(29.3)$ & $10(22.2)$ & 0.36 & $37(25.9)$ & $9(36.0)$ & 0.295 \\
\hline $\begin{array}{l}\text { Alleviate disease symptoms/toxic effect of } \\
\text { conventional therapy }\end{array}$ & $44(26.2)$ & $29(23.6)$ & $15(33.3)$ & 0.2 & $36(25.2)$ & $8(32.0)$ & 0.474 \\
\hline Improve psychological well-being and finding hope & $30(17.9)$ & $21(17.1)$ & $9(20.0)$ & 0.66 & $27(18.9)$ & $3(12.0)$ & 0.574 \\
\hline $\begin{array}{l}\text { More compatible with own value/belief towards } \\
\text { life and health }\end{array}$ & $26(15.5)$ & $22(17.9)$ & $4(8.9)$ & 0.15 & $24(16.8)$ & $2(8.0)$ & 0.374 \\
\hline Allow to relax and helps in sleep & $24(14.3)$ & $20(16.3)$ & $4(8.9)$ & 0.23 & $20(14.0)$ & $4(16.0)$ & 0.76 \\
\hline Seeking a cure & $23(13.7)$ & $17(13.8)$ & $6(13.3)$ & 0.94 & $19(13.3)$ & $4(16.0)$ & 0.753 \\
\hline Complementary effect to conventional medicine & $21(12.5)$ & $15(12.2)$ & $6(13.3)$ & 0.84 & $16(11.2)$ & $5(20.0)$ & 0.207 \\
\hline More control over own treatment & $15(8.9)$ & $11(8.9)$ & $4(8.9)$ & 1 & $12(8.4)$ & $3(12.0)$ & 0.471 \\
\hline Conventional treatment is too toxic & $7(4.2)$ & $6(4.9)$ & $1(2.2)$ & 0.68 & $6(4.2)$ & $1(4.0)$ & 1 \\
\hline Dissatisfied with conventional medicine & $4(2.4)$ & $3(2.4)$ & $1(2.2)$ & 1 & $4(2.8)$ & 0 & 1 \\
\hline
\end{tabular}

Columns do not sum to $100 \%$ due to the option of multiple answers.

$\mathrm{P}^{1}$ comparison of patients with malignant verses benign tumours.

$\mathrm{P}^{2}$ comparison of adult verses paediatric patients.

noted was that patients with benign tumour were more likely to start CAM resulting from their own initiative. Majority (68.5\%) of CAM users did not discuss these therapies with their doctors. The non-disclosure rates were similar for patients with malignant verses benign tumors $(p=0.941)$ and for adult verses paediatric patients $(p=1)$. The 3 most common reasons for non-disclosure were; 'felt it was unnecessary' (42.6\%), 'doctors did not ask' (35\%) and 'afraid to tell as doctor may not agree' (10.6\%). For patients who did disclose, the doctors responded as follows; remained neutral (51\%), encouraged (35.8\%) and discouraged (13.2\%) CAM use. Only $25.6 \%$ of patients were proactively asked about their CAM usage by their doctors.

\section{Discussion}

To our best knowledge, this study is the first to specifically explore CAM usage amongst patients with orthopaedic oncology tumours. Since there have been no other similar studies, we related our findings to other comparable studies, depending on the context of discussion. The prevalence of CAM used amongst orthopaedic oncology patients was high. Overall, 61.3\% patients used at least one CAM modality during their current illness. CAM usage was significantly higher amongst patients with malignant tumours compared with their benign tumours counterparts $(68 \%$ versus $48.4 \%, \mathrm{p}=0.002)$; concurring with a nationwide survey amongst Japanese cancer patients which displayed higher CAM usage in malignant tumours compared with benign tumours; albeit at lower rates (45\% versus $26 \%$ ) [26].The rate of $68 \%$ noted amongst patients with malignant tumours was higher than the prevalence reported from previous studies amongst cancer patients [6,10,18,26-28]. In a large European survey [5], the highest prevalence of CAM was in pancreatic (56.3\%), liver (55.6\%), bone/spinal (54.5\%) and brain cancer

Table 4 CAM modalities used amongst 168 CAM users

\begin{tabular}{|c|c|c|c|c|c|c|c|}
\hline Modalities & $\begin{array}{l}\text { All patients } \\
(\mathrm{N}=168)(\%)\end{array}$ & $\begin{array}{l}\text { Malignant } \\
(\mathrm{N}=123)(\%)\end{array}$ & $\begin{array}{l}\text { Benign } \\
(N=45)(\%)\end{array}$ & $P^{1}$ & $\begin{array}{l}\text { Adults } \\
(\mathrm{N}=143)(\%)\end{array}$ & $\begin{array}{l}\text { Paediatric } \\
(\mathrm{N}=25)(\%)\end{array}$ & $\mathrm{P}^{2}$ \\
\hline Biological & $152(90.5)$ & $115(93.5)$ & $37(82.2)$ & 0.04 & $130(90.9)$ & $22(88.0)$ & 0.710 \\
\hline Mind-body & $68(40.5)$ & $47(38.2)$ & $21(46.7)$ & 0.32 & $61(42.7)$ & 7 (28.0) & 0.168 \\
\hline Physical & $36(21.4)$ & 19 (15.4) & 17 (37.8) & 0.002 & $33(23.1)$ & $3(12.0)$ & 0.213 \\
\hline Alternative & $24(14.3)$ & 18 (14.6) & $6(13.3)$ & 0.83 & $24(16.8)$ & $0(0)$ & 0.027 \\
\hline Energy & $15(8.9)$ & $12(9.8)$ & $3(6.7)$ & 0.76 & $13(9.1)$ & $2(8.0)$ & 1 \\
\hline
\end{tabular}

Columns do not sum to $100 \%$ due to the option of multiple answers.

$\mathrm{P}^{1}$ comparison of patients with malignant verses benign tumours.

$\mathrm{P}^{2}$ comparison of adult verses paediatric patients. 
Table 5 Types of CAM used amongst 168 CAM users

\begin{tabular}{|c|c|c|}
\hline CAM & $\mathbf{N}$ & $\%$ \\
\hline \multicolumn{3}{|l|}{ Biological therapy } \\
\hline \multicolumn{3}{|l|}{ Vitamin and mineral supplements } \\
\hline Mega/multivitamins & 52 & 31 \\
\hline Calcium suplements & 12 & 7 \\
\hline Antioxidant pills & 7 & 4.2 \\
\hline \multicolumn{3}{|l|}{ Herbal } \\
\hline Sea cucumber/gamat (Stichopus horrens) & 30 & 18 \\
\hline Herbal remedies & 22 & 13 \\
\hline Fruit juice with antioxidant properties & 17 & 10 \\
\hline Spirulina (Arthrospira plantensis) & 15 & 9 \\
\hline Belalai gajah/sabah snake grass (Clinacanthus nutans) & 13 & 7.7 \\
\hline Other medicinal plant & 9 & 5.4 \\
\hline Medicinal mushroom supplements & 9 & 5.4 \\
\hline Aloe vera & 8 & 4.8 \\
\hline Apricot seed & 7 & 4.2 \\
\hline Ginseng & 7 & 4.2 \\
\hline Evening primrose & 6 & 3.6 \\
\hline Green tea & 6 & 3.6 \\
\hline Habatus sauda seeds & 6 & 3.6 \\
\hline Medicinal tea & 5 & 3 \\
\hline Ginko biloba & 5 & 3 \\
\hline \multicolumn{3}{|l|}{ Non-herbal } \\
\hline Snakehead/haruan (Chana striatus) & 47 & 28 \\
\hline Fish oil & 22 & 13 \\
\hline Porcupine quill powder/dates & 8 & 4.8 \\
\hline Honey & 6 & 3.6 \\
\hline Special water & 5 & 3 \\
\hline Birds nest & 5 & 3 \\
\hline Special diet & 10 & 6 \\
\hline \multicolumn{3}{|l|}{ Physical therapy } \\
\hline Massage therapy & 33 & 19.6 \\
\hline Reflexology & 2 & 1.2 \\
\hline Chiropractic & 1 & 0.6 \\
\hline Cupping & 2 & 1.2 \\
\hline \multicolumn{3}{|l|}{ Mind-body medicine } \\
\hline Prayer/faith healing & 52 & 31 \\
\hline Holy water/zam zam water & 22 & 13 \\
\hline Traditional healers & 9 & 5.4 \\
\hline Meditation & 8 & 4.8 \\
\hline Yoga & 4 & 2.4 \\
\hline Support group & 4 & 2.4 \\
\hline Mental imagery & 1 & 0.6 \\
\hline
\end{tabular}

Table 5 Types of CAM used amongst 168 CAM users (Continued)

\begin{tabular}{|c|c|c|}
\hline Energy therapy & & \\
\hline Bioelectromagnetic therapy & 7 & 4.2 \\
\hline Qi Gong & 3 & 1.8 \\
\hline Manual healing & 2 & 1.2 \\
\hline Taichi & 2 & 1.2 \\
\hline Reiki & 1 & 0.6 \\
\hline Ionic foot bath & 1 & 0.6 \\
\hline Longevitology & 1 & 0.6 \\
\hline \multicolumn{3}{|l|}{ Alternative therapy } \\
\hline Acupuncture & 16 & 9.5 \\
\hline Traditional Chinese medicine & 10 & 6 \\
\hline Traditional Indian medicine & 2 & 1.2 \\
\hline Homeopathy & 2 & 1.2 \\
\hline
\end{tabular}

totals are thus greater than total participants.

(50\%) patients. However, the number of bone/spinal cancer patients was small $(\mathrm{n}=22)$, representing $2.3 \%$ of patients in this series.

Apart from malignant tumours, the other independent predictors for CAM use were paediatric patients and patients who underwent surgery. A considerable proportion of paediatric patients used CAM, with significantly higher rates than adults $(78 \%$ versus $59 \%, \mathrm{p}=0.04)$. Likewise, high CAM usage (84.5\%) was noted in another study involving Malaysian children with cancer [20]. In a systematic review, CAM prevalence amongst children with cancers ranged from $6 \%$ to $91 \%$ [19]. This has been linked to lower survival perspective and fewer days since relapse [29], suggesting that CAM therapies may act as coping strategies for families in efforts to try everything

Table 6 Source of information amongst 168 CAM users

\begin{tabular}{|c|c|c|c|c|}
\hline Source & $\begin{array}{l}\text { Total } \\
(\mathrm{N}=168)(\%)\end{array}$ & $\begin{array}{l}\text { Malignant } \\
(\mathrm{N}=123)(\%)\end{array}$ & $\begin{array}{l}\text { Benign } \\
(\mathrm{N}=45)(\%)\end{array}$ & $\mathrm{p}$ \\
\hline $\begin{array}{l}\text { From family } \\
\text { members }\end{array}$ & $89(53.0)$ & $61(49.6)$ & $28(62.2)$ & 0.15 \\
\hline From friends & $82(49.0)$ & $63(51.6)$ & $19(42.2)$ & 0.28 \\
\hline $\begin{array}{l}\text { From mass media/ } \\
\text { websites }\end{array}$ & $40(24.0)$ & $28(23.0)$ & $12(26.7)$ & 0.62 \\
\hline Your own free will & $31(18.5)$ & $16(13.0)$ & $15(33.3)$ & 0.003 \\
\hline $\begin{array}{l}\text { From health } \\
\text { personnel }\end{array}$ & $24(14.3)$ & $18(14.6)$ & $6(13.3)$ & 0.83 \\
\hline From other patients & $20(12.0)$ & $15(12.3)$ & $5(11.1)$ & 0.83 \\
\hline $\begin{array}{l}\text { From CAM } \\
\text { practitioner }\end{array}$ & $16(9.5)$ & 13 (10.6) & $3(6.7)$ & 0.56 \\
\hline $\begin{array}{l}\text { From your church/ } \\
\text { religious group }\end{array}$ & $5(3.0)$ & $3(2.4)$ & $2(4.4)$ & 0.61 \\
\hline
\end{tabular}

Columns do not sum to $100 \%$ due to the option of multiple answers. 
possible to cure or alleviate the pain and distress in their children. Although we did not determine the effects of parenteral socio-demographic and education characteristics on CAM practices in children, no such influence was found in another study involving Malaysia children with cancer [20]. Children whose parents used CAM were more likely to use CAM compared to children whose parents did not [30]; this is not unexpected since children are dependent on their parents for their healthcare decisions.

Concurring with another study [7], patients who underwent surgery for tumour removal were more likely to use CAM, particularly various biological modalities. This finding should not be underestimated, as clinical complications during perioperative period can result from natural therapies, either from direct effect or herb-drug interaction [17]. Thus, patients scheduled for surgery should be questioned about none prescription medications usage [17]. In retrospect, we should have added a question regarding CAM timing in relation to the surgery.

Patients with metastasis and those who received chemotherapy were more likely to use CAM, although these factors were not predictive in multivariate analysis. While some studies reveal higher CAM usage amongst patients with metastatic cancers [18], others suggest no association with advanced disease $[7,20]$. Stage of disease impacted patients' reasons and expectations for CAM usage, although it may not predict CAM use [7]. Although higher education and socio-economic status have been linked to CAM usage $[1,5,8,18]$; concurring with other studies we found no such association [7]. Reasons that catalyze CAM use in countries with higher socio-economic status, might work in opposite directions in lower socio-economic status countries [8]; the latter attributed to cultural factors and more traditional lifestyle.

It is important for doctors to recognize the reasons motivating patients' CAM usage. Our patients were mainly motivated by the desire to try out everything possible to fight the disease, to improve their general health and to enhance wound healing. The first two reasons emerged at significantly higher rates in patients with malignant compared with benign tumours, suggesting that these patients may be turning to CAMs as additional measures to enhance general overall health, rather than focusing solely on their illness; supporting the philosophical congruence theory [1]. CAM use was also fuelled by the desire to control disease progression, boost the immune system and to cope with treatment and/or its side-effects. Reassuringly, only 4 patients attributed dissatisfaction with conventional medicine as a reason for using CAM. Our findings indicate that patients are not deserting conventional therapies which have been thoroughly tested, for unconventional treatment which lack scientific evidence regarding safety and efficacy.

Due to the physical and psychological impacts of an oncology condition, coping strategies are essential tools for patients and parents to move through the illness trajectory. Both biological therapies and mind-body techniques were most frequently used amongst our patients and its use may be explained by Lazarus and Folkmans' problem-focused and emotion-focused coping strategies $[31,32]$. Problem-focused strategies include the use of various biological therapies in attempts to alleviate diseaserelated symptoms or adverse-effects of conventional treatment, while prayers and meditation techniques are examples of emotional-focused coping strategies as patients venture into a spiritual and emotional journey [18]. Factors that drive patients and parents towards the uptake of CAM may also be viewed from a problemfocused verses emotion -focused perspective. Overall, when the factors motivating CAM usage were analysed, they were skewed towards problem-focused factors $(>20 \%$ frequency each); these included enhancing physical wellbeing, accelerating wound-healing, controlling disease progression, boosting immune system and ameliorating symptoms resulting from disease and treatment. Parents of paediatric oncology patients also seemed lop-sided towards problem-focused factors. During conventional treatment, parents often play a passive role with little opportunity for active involvement in the healing process of their children [33]. Coping strategies focusing on problemsolving are action-centred and enables more involvement in care activities of their child [33]. Other reasons such as improving psychological well-being and finding hope, taking more control over own treatment, allowing to relax/ sleep may be described as emotion-focused factors and were less frequently mentioned $(<20 \%$ frequency) in our study.

Majority of our patients were satisfied with CAM usage and would continue its use and even recommend to others. We believe that the satisfaction shown was because the expectations for CAM were met. Majority of our patients took CAM with the hope of enhancing their physical wellbeing and healing process, whilst only a small proportion took CAM with the intention to cure their illness. This mirrors the reasons for taking CAM and the resulting satisfaction amongst Singaporean cancer patients [27]. Conversely, disappointment towards CAM was high amongst Nigerian cancer patients, as majority used CAM to treat or cure cancer, with unmet expectation ultimately [34].

Biological-based therapies were the most common CAM used, with vitamins, snakehead and sea cucumber, constituting the three most common biological therapies. Vitamins were widely used in multiple series $[5,7,27]$, reflecting self-promoting behaviour contributing to general health. A substantial proportion of our patients embarked on various prayer healing methods and almost all patients used these techniques alongside other CAM modalities. Prayers and spiritual therapies are important aspects amongst Malaysian oncology patients [35] and 
strong connections between reliance on religious beliefs and the ability to cope with cancer has been shown [3], contributing to psychosocial adjustment to cancer and its treatment.

While the variances of what constitutes CAM possibly contribute to the diverse prevalence rates reported in literature, we believe the multi-ethnicity of our patients with its diverse cultural background, religious beliefs and practices contribute to the high prevalence and differential patterns of CAM use. Worldwide these practices differ depending on cultural habits, geographic locations, ethnic backgrounds and religious beliefs. Majority of Japanese cancer patients used products such as mushrooms, herbs and shark cartilage [26], whereas spiritual practices and vitamins which were widespread in our study and Western countries [7] were rarely used amongst the Japanese cancer population [26]. Singapore cancer patients commonly used traditional Chinese medicine, bird's nest and special diets [27], whereas in Turkey, herbal therapies such as stinging nettle were used widely [28]. While spiritual approaches, vitamins and herbs were the most common CAMs used in USA [7], Australian cancer patients used mainly dietary supplements, prayers, herbs and relaxation techniques/meditation [18]. Generally, natural products are widely used amongst cancer patients emphasizing an appeal for 'natural' remedies, as many patients believe natural equates to safe.

While energy, alternative and mind-body medicine had similar usage across patients with benign and malignant tumor, biological therapies were more common amongst patients with malignant tumors. Contrariwise, physical therapies involving various massage therapies were more common amongst patients with benign tumor. Although massage therapies alleviate anxiety and distress symptoms amongst cancer patients, rare complications such as haemorrhage and fractures can occur [36], especially with pre-existing weakened bone from underlying pathologies. Acupuncture used by $10 \%$ of our patients, has been shown to relieve cancer related pain and chemotherapy induced nausea and vomiting [37]. However, it should be used cautiously in patients with deranged clotting mechanisms e.g. with impaired liver function, as bleeding can occur [37]. Only $2.4 \%$ of our patients used support groups, suggesting a lack of support group amongst patients with musculoskeletal tumours.

Children are more likely to use biologically-based therapies and mind-body therapies compared with alternative or energy therapies [30], concurring with the findings of our study. In a study conducted amongst children and adolescents with cancer, adolescents showed a tendency towards using more invasive therapies (e.g., acupuncture) compared to children [38].

Majority of the patients seemed to detach CAM and conventional medicine by not disclosing CAM use to their doctors, with most patients having the perception that disclosure was unnecessary. Disclosure rates of $20 \%$ to $77 \%$ have been reported [39] and the main reasons for non-disclosure were doctor's lack of inquiry; patient's anticipation of the doctor's disapproval, disinterest, or inability to help and patient's perception that CAM disclosure is irrelevant to conventional care [39].

Our study supports the role of family members and friends on patient's CAM uptake. Significant others (SOs) such as family members, partners and close friends influence decisions about CAM use amongst cancer patients, often acting as information providers and active decision makers $[40,41]$. Qualitative studies also indicate the importance of a supportive social network and the involvement of SOs in uptake and maintenance of CAM, contributing to bonding between patients and their SOs [41].

Many patients relied on information from unscientific sources, such as mass media and internet. However, these patients may be at risk of adverse effects as many websites promote CAM without scientifically proven safety and efficacy data [42]. Only $14.3 \%$ of our patients received information regarding CAM from healthcare professionals. This may be due to the fact that most healthcare professionals feel they lack adequate knowledge or were not up-to-date with the best evidence on CAM use in oncology [43].

Few limitations of this study must be acknowledged. The drawback of face-to-face compared to anonymous questionnaire is that participants may not disclose certain information for fear of negative repercussions. However, face-to-face interview allows respondents and interviewers to seek clarification, increasing the response rates and reliability of answers. Secondly, although we did attempt to provide an exhaustive list of CAMs to ensure consistency in defining CAMs and to reduce recall bias; participants might still have been less likely to report the non-listed CAMs. Although this study was confined to a single institution, being one of the main orthopaedic oncology referral centre in this country, it receives majority of referrals in this region. Thus, this study could be taken as a proxy indicator for CAM usage amongst orthopaedic oncology patients.

\section{Conclusions}

This study highlights a high prevalence of CAM usage amongst orthopaedic oncology patients, especially amongst patients with malignant tumors, paediatric patients and patients who undergo surgery. Patients appear to use CAM in conjunction with, not instead of conventional medicine. Biological-based therapies were the most popular CAM therapies used. Majority patients used CAM with the hope of enhancing their physical well-being and healing process and were generally satisfied with the CAMs. Given the high prevalence of CAM usage amongst orthopaedic oncology 
patients, oncologists should proactively ask their patients about CAM usage as most patients do not voluntarily disclose this information. In order to foster better patientdoctor communication, oncologists should be familiar with the commonly used CAM amongst patients, or at least be able to direct them to reliable sources of information.

\section{Competing interests}

The authors declare that they have no competing interests.

\section{Authors' contributions}

AD conceived of the study, participated in its design, analysed of data, and wrote-up the manuscript. YTZ, SYJL, ILSZ contributed to the conceptualization of the study, coordinated the data collection and participated in data analysis. VAZ contributed to the conceptualization of the study, co-supervised data collection and contributed to the manuscript write-up. All authors read and approved the final manuscript.

\section{Acknowledgement}

We wish to acknowledge Venotha Madhialogan for her significant contribution in data collection.

\section{Author details}

${ }^{1}$ Jeffrey Cheah School of Medicine and Health Sciences, Monash University, 47500 Bandar Sunway, Malaysia. ${ }^{2}$ Department of Orthopaedic Surgery (Noceral), University of Malaya Medical Centre, 59100 Kuala Lumpur, Malaysia.

Received: 25 July 2014 Accepted: 22 September 2014

Published: 17 October 2014

\section{References}

1. Astin JA: Why patients use alternative medicine: results of a national study. JAMA 1998, 279(19):1548-1553.

2. Saydah SH, Eberhardt MS: Use of complementary and alternative medicine among adults with chronic diseases: United States 2002. J Altern Complement Med 2006, 12(8):805-812.

3. Weaver AJ, Flannelly $\mathrm{KJ}$ : The role of religion and spirituality for cancer patients and their caregivers. South Med J 2004, 97(12):1210-1214.

4. National Center for Complementary and Alternative Medicine: What is Complementary and Alternative Medicine (CAM)? https://cim.ucdmc ucdavis.edu/clubs/camsig/whatiscam.pdf last accessed 15 September 2014.

5. Molassiotis A, Fernadez-Ortega P, Pud D, Ozden G, Scott JA, Panteli V, Margulies A, Browall M, Magri M, Selvekerova S, Madsen E, Milovics L, Bruyns I, Gudmundsdottir G, Hummerston S, Ahmad AM, Platin N, Kearney N, Patiraki E: Use of complementary and alternative medicine in cancer patients: a European survey. Ann Oncol 2005, 16(4):655-663.

6. Swisher EM, Cohn DE, Goff BA, Parham J, Herzog TJ, Rader JS, Mutch DG: Use of complementary and alternative medicine among women with gynaecologic cancers. Gynecol Oncol 2002, 84(3):363-367.

7. Richardson MA, Sanders T, Palmer JL, Greisinger A, Singletary SE: Complementary and alternative medicine use in a comprehensive cancer center and the implications for oncology. J Clin Oncol 2000, 18(13):2505-2514.

8. Verhoef MJ, Balneaves LG, Boon HS, Vroegindewey A: Reasons for and characteristics associated with complementary and alternative medicine use among adult cancer patients: a systematic review. Integr Cancer Ther 2000, 4(4):274-286.

9. Ernst $E$, Cassileth BR: The prevalence of complementary/alternative medicine in cancer: a systematic review. Cancer 1998, 83(4):777-782.

10. Horneber M, Bueschel G, Dennert G, Less D, Ritter E, Zwahlen M: How many cancer patients use complementary and alternative medicine: a systemic review and metaanalysis. Integr Cancer Ther 2012, 11(3):187-203.

11. Molassiotis A, Potrata B, Cheng KK: A systematic review of the effectiveness of Chinese herbal medication in symptom management and improvement of quality of life in adult cancer patients. Complement Ther Med 2009, 17(2):92-120.

12. Liossi $C$, White $P$, Hatira $P$ : A randomized clinical trial of a brief hypnosis intervention to control venepuncture-related pain of paediatric cancer patients. Pain 2009, 142(3):255-263.
13. Oh B, Butow P, Mullan B, Hale A, Lee MS, Guo X, Clarke S: A critical review of the effects of medical Qigong on quality of life, immune function, and survival in cancer patients. Integr Cancer Ther 2012, 11(2):101-110.

14. Markman M: Safety issues in using complementary and alternative medicine. J Clin Oncol 2002, 20(18 Suppl):39S-41S.

15. Markowitz JS, Donovan JL, DeVane CL, Taylor RM, Ruan Y, Wang JS, Chavin KD: Effect of St John's wort on drug metabolism by induction of cytochrome P450 3A4 enzyme. JAMA 2003, 290(11):1500-1504.

16. Anon. From the Centers for Disease Control and Prevention: Hepatic toxicity possibly associated with kava-containing products-United States, Germany, and Switzerland, 1999-2002. JAMA 2003, 289(1):36-37.

17. Ang-Lee MK, Moss J, Yuan CS: Herbal medicines and perioperative care. JAMA 2001, 286(2):208-216.

18. Klafke N, Eliott JA, Wittert GA, Olver IN: Prevalence and predictors of complementary and alternative medicine (CAM) use by men in Australia cancer ouptatients services. Ann Oncol 2012, 23(6):1571-1578.

19. Bishop FL, Prescott P, Chan YK, Saville J, von Elm E, Lewith GT: Prevalence of complementary medicine use in paediatric cancer: a systematic review. Pediatrics 2010, 125(4):768-776.

20. Hamidah A, Rustam ZA, Tamil AM, Zarina LA, Zulkifli ZS, Jamal R: Prevalence and parental perceptions of complementary and alternative medicine use by children with cancer in a multi-ethnic Southeast Asian population. Pediatr Blood Cancer 2009, 52(1):70-74.

21. Pui CH, Gajjar AJ, Kane JR, Qaddoumi IA, Pappo AS: Challenging issues in paediatric oncology. Nat Rev Clin Oncol 2011, 8(9):540-549.

22. Ladas EJ, Post-White J, Hawks R, Taromina K: Evidence for symptom management in the child with cancer. J Pediatr Hematol Oncol 2006, 28(9):601-615.

23. Fletcher CDM, Bridge JA, Hogendoorn PCW, Mertens F: World Health Organization Classification of tumours of soft tissue and bone. 4th edition. Lyon: IARC Press; 2013

24. National Center for Complementary and Alternative Medicine: Expanding Horizons of Healthcare: Five-Year Strategic Plan, 2001-2005. http:// nccam.nih.gov/sites/nccam.nih.gov/files/about/plans/fiveyear/fiveyear.pd (last accessed 15 September 2014).

25. Wieland LS, Manheimer E, Berman BM: Development and classification of an operational definition of complementary and alternative medicine for the Cochrane collaboration. Altern Ther Health Med 2011, 17(2):50-59.

26. Hyodo I, Amano N, Eguchi K, Narabayashi M, Imanishi J, Hirai M, Nakano T, Takashima S: Nationwide survey on complementary and alternative medicine in cancer patients in Japan. J Clin Oncol 2005, 23(12):2645-2654.

27. Shih V, Chiang JY, Chan A: Complementary and alternative medicine (CAM) usage in Singaporean adult cancer patients. Ann Oncol 2009, 20(4):752-757.

28. Yildiz I, Ozguroglu M, Toptas T, Turna H, Sen F, Yildiz M: Patterns of complementary and alternative medicine use among Turkish cancer patients. J Palliat Med 2013, 16(4):383-390.

29. Tomlinson D, Hesser T, Ethier MC, Sung L: Complementary and alternative medicine use in pediatric cancer reported during palliative phase of disease. Support Care Cancer 2011, 19(11):1857-1863.

30. Barnes PM, Bloom B, Nahin RL: Complementary and alternative medicine use among adults and children: United States, 2007. Natl Health Stat Report 2008, 12:1-23.

31. Lazarus RS, Folkman S: Stress, Appraisal, and Coping. New York: Springer; 1984

32. Folkman S, Lazarus RS: Coping as a mediator of emotion. J Pers Soc Psychol 1988, 54(3):466-475.

33. LaMontagne LL, Hepworth JT, Pawlak R, Chiafery M: Parental coping and activities during pediatric critical care. Am J Crit Care 1992, 1(2):76-80.

34. Ezeome ER, Anarado AN: Use of complementary and alternative medicine by cancer patients at the University of Nigeria Teaching Hospital, Enugu, Nigeria. BMC Complement Altern Med 2007, 7:28.

35. Farooqui M, Hassali MA, Shatar AK, Shafie AA, Seang TB, Farooqui MA: A qualitative exploration of Malaysian cancer patients' perspectives on cancer and its treatment. BMC Public Health 2011, 11:525.

36. Corbin L: Safety and efficacy of massage therapy for patients with cancer. Cancer Control 2005, 12(3):158-164.

37. Lin JG, Chen $\mathrm{YH}$ : The role of acupuncture in cancer supportive care. Am J Chin Med 2012, 40(2):219-229.

38. Gottschling S, Meyer S, Längler A, Scharifi G, Ebinger F, Gronwald B: Differences in use of complementary and alternative medicine between 
children and adolescents with cancer in Germany: a population based survey. Pediatr Blood Cancer 2014, 61(3):488-492.

39. Davis EL, Oh B, Butow PN, Mullan BA, Clarke S: Cancer patient disclosure and patient-doctor communication of complementary and alternative medicine use: a systematic review. Oncologist 2012, 17(11):1475-1481.

40. Öhlén J, Balneaves $L G$, Bottorff JL, Brazier ASA: The influence of significant others in complementary and alternative medicine decisions by cancer patients. Soc Sci Med 2006, 63(6):1625-1636.

41. Klafke N, Eliott JA, Olver IN, Wittert GA: The role of complementary and alternative medicine (CAM) routines and rituals in men with cancer and their significant others (SOs). A qualitative investigation. Support Care Cancer 2014, 22(5):1319-1331.

42. Schmidt K, Ernst E: Assessing websites on complementary and alternative medicine for cancer. Ann Oncol 2004, 15(5):733-742.

43. Chang KH, Brodie R, Choong MA, Sweeney KJ, Kerin MJ: Complementary and alternative medicine use in oncology: a questionnaire survey of patients and health care professionals. BMC Cancer 2011, 11:196.

doi:10.1186/1472-6882-14-404

Cite this article as: Dhanoa et al:: Complementary and alternative medicine use amongst Malaysian orthopaedic oncology patients. BMC Complementary and Alternative Medicine 2014 14:404.

\section{Submit your next manuscript to BioMed Central and take full advantage of:}

- Convenient online submission

- Thorough peer review

- No space constraints or color figure charges

- Immediate publication on acceptance

- Inclusion in PubMed, CAS, Scopus and Google Scholar

- Research which is freely available for redistribution 